Volume 9, No.4, July - August 2020

International Journal of Advanced Trends in Computer Science and Engineering

Available Online at http://www.warse.org/IJATCSE/static/pdf/file/ijatcse311942020.pdf

https://doi.org/10.30534/ijatcse/2020/311942020

\title{
IoT Enhanced Smart Mirror for Personal and Commercial Applications
}

\author{
P.Selvaraj $^{1}$, Lakshmi Kanthan Narayanan ${ }^{2}$ \\ ${ }^{1}$ SRM Institute of Science and Technology, Kattankulathur, Tamilnadu, India, selvarap@ srmist.edu.in \\ ${ }^{2}$ Chennai Institute of Technology, Tamilnadu, India, lakshmikanthann@ @itchennai.net
}

\begin{abstract}
The computing capability of the cloud computing drives the IoT applications to the level applicable in the real time based use cases, with the support of more immersive and conducive UI based interactions. The evolution of Internet of Things facilitates variety of home based and commercial systems with advent multi-dimensional application independency. A smart mirror is one in every of the recent inseparable smart home decorative product. In this work, a cost effective smart mirror was developed and designed on the RPI's Debian based Raspbian Operating System platform and projected over the display interface with the assistance of Apache web server. It fetched updated data from the ThingSpeak cloud platform for the users. It was made up of a standard mirror and a projection unit. It prevalently used to display real time updates and prompt notifications like weather, traffic, calendar, news feeds, maps and social media with an intelligent open-ended voice search interface. It also used updates in a more immersive manner. It was controlled the net technologies like PHP, java script and Cascaded style Sheet for the designing of interfaces and server side maneuvers. It completely run through the PHP scripts with basically no complex external dependencies. Many open source API's like Google maps API, Google $\mathrm{Cal}$ and open weather chart API were used for gathering information from internet. Several performance evaluations had been done to justify the intelligent smart mirror as an amenable product for the personal as well as commercial use.
\end{abstract}

Key words: Cloud, Internet of Things, Raspberry Pi3, Smart Mirror.

\section{INTRODUCTION}

The different technologies have been invented for the betterment of the human lives over the past years. The combination of technologies that emulates the near human behaviors, has invaded into the people's life style and become the newest trend. One such thing is that the Internet of Things (IoT), which is a briefing of interrelated devices with the capacity to send information over a system without expecting $\mathrm{H} 2 \mathrm{H}$ (Human 2 Human) or H2M (Human 2 Machine) collaboration [1]. The IoT, is utilized in different applications like implanting a biochip transponder in man for the higher heart monitoring and a vehicle that notifies the driving force when something goes wrong. The IoT has been developed from the mixture of remote advances, smaller scale electromechanical (MEMS) frameworks, miniaturized scale administrations and therefore the Internet. The smart mirror is an inspiration of integrating IoT based technology into the conventional mirror. Despite the actual fact that any information may often accessible on the user's mobile and other hand held devices, it is time-saving and convenient to urge the knowledge available within the mirror during the everyday bathroom routines. The efficiency and performance are increasingly establishing their dominance as selling points and companies had started using and promoted their products. The very fact that any product manufacturer competes or striving to extend is that the performance. This work has focused on improving the performance of the smart mirror through the cost effective solution. The performance evaluations have been done to analyze the behavior of the proposed intelligent smart mirroring system.

\section{EARLIER WORKS ON SMART MIRROR}

In 2014, Toshiba [2] had built up a TV based on the idea of smart interaction including touch functionalities M. Anwar Hossain et al., [3] discussed the structure and advancement of an innovative smart mirror that speaks to a subtle interface for the surrounding home condition. The smart mirrors were accustomed to provide a characteristic methods for connection through which the inhabitants can control and access the user's keen apparatuses through the customized administrations.

The smart mirror functionalities are shown by build up an effortlessly extendable home mechanization framework that encourages the combo of clan machines and different altered 
data administrations. Emphasis is additionally given to ensure accommodation in reaching to these administrations with a base measure of client mediation. As an example, confront acknowledgment based verification was utilized to naturally recognize the client confronting the mirror and given

gadget based interface to urge information with different administrations. Administration situated engineering has been embraced to form and convey the various administrations, where the mirror interface, the apparatuses, and also the news and knowledge channels were created with an online benefit correspondence systems.

Fujinami K and Kawsar [4] discussed about sending both an image and data to the smart clients. The creative mirror enabled a client to audit electronic data, while performing individual cleanliness methods. As an example, the client could take a gander at the innovative mirror and audit news features further as stories, read and react to messages, or potentially survey and alter their calendar of arrangements, while prepping. The mirror had two modes. In an exceedingly power off mode, the smart mirror went about as a typical intelligent mirror. In a very power on mode, at that time the smart mirror was a showcase gadget. The presentation gadget involved slight screen, which might permit coordinate client interaction [5].

\section{SMART MIRROR AND ITS EXPECTATIONS}

This section covers a good range of issues which may be faced from the stage of putting in the smart mirror to using it on an each day routines. The requirement of this device would be a discovery where the technology and therefore the ordinary elements of the human life to merge and supply a far better experience. The feasible uses of this device depend upon the quantity of services the user expects from the merchandise. Generally the smart mirrors are used to know the time, weather outlook, social media greetings, and calendar based notifications [6].

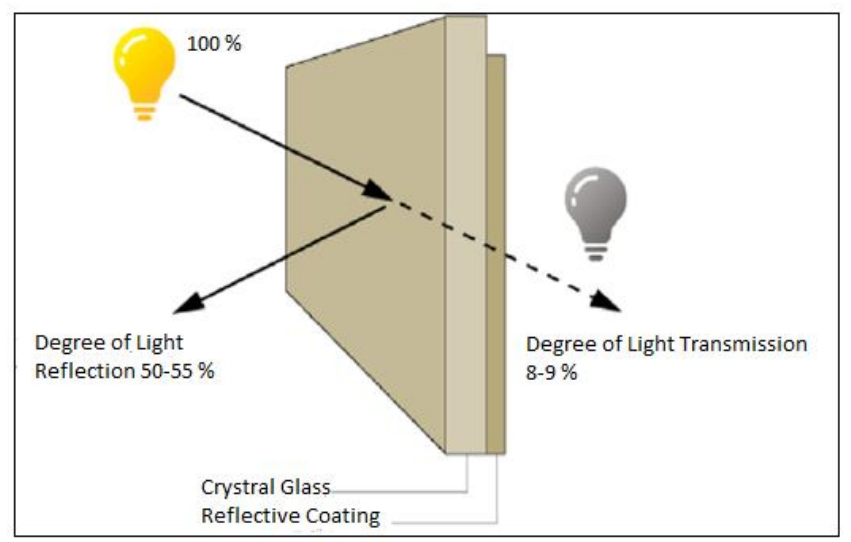

Figure 1: Two Way Mirror and the degree of light reflection
A restricted mirror is often utilized as a clearly ordinary mirror in an exceedingly brilliantly lit room, with a plenty darker room on the other side considering the physical properties of the light waves (Figure.1). Individuals on the splendidly lit side can see their own reflection and it would appear as a standard mirror. Individuals on the clouded side transparently appear as if a simple window. The sunshine from the brilliant room reflected from the mirror another time into the area itself may be a lot more noteworthy than the sunshine transmitted from the dull room, overpowering the small measure of sunshine transmitted from the dull to the brilliant room. Then again, the sunshine reflected yet again into the clouded side is overpowered by the sunshine transmitted from the brilliant side. This allows a watcher in obscurity side to observe the brilliant room secretly.

At the purpose when such mirrors are utilized for one-way perception, the survey room is kept dull by an obscured window ornament or a twofold entryway vestibule. These perceptions on dark rooms have been utilized in the following scenarios:

- Execution rooms

- Experimental research

- Police interrogation rooms

- Market investigations

- Security perception decks in the open territories. The analysis of the proposed smart mirror implementation comprises of functional and non-functional requirements. The following are the functional requirements of the smart mirror:

1. Identifying the customer needs

2. Analyzing the feasibility factors

3. Finding the system definitions.

Hence the following requirements were defined. 1. Features of the system should fulfill the purpose of the system. 2. The major focus should be on the customer needs and their problems with respect to the smart mirror. 3. The functionality required to satisfy user's demands and making their life easy by enhancing the way of delivering the content.

\section{SYSTEM DESIGN}

To develop a sensible mirror design with the user's expectation a big research had been done. First, similar projects and products were investigated to grasp the very fact what has been done and possibly what must be done in a conventional sense when considering the form factors. Then the individual components of the mirror had been analyzed so as to make a decision on the expected features of the merchandise. The design of the smart mirror was dampened into subsystems to represent important components of the project. Since there are hardware and software components to style, a diagram plan was needed for both. The technical specifications were written for every subsystem during the prototyping stage. 


\subsection{Hardware Design}

The hardware component mainly comprises of the Raspberry pi and also the display device (Tablet) together with the 2-way mirror. The voice recognition was exploited with the in-built microphone of the tablet getting used to acknowledge the voice commands being issued by the user. These commands were taken in as input and supported the pre-defined functions the required functions like maps or calendar were executed and displayed. This design is shown in Figure 2.

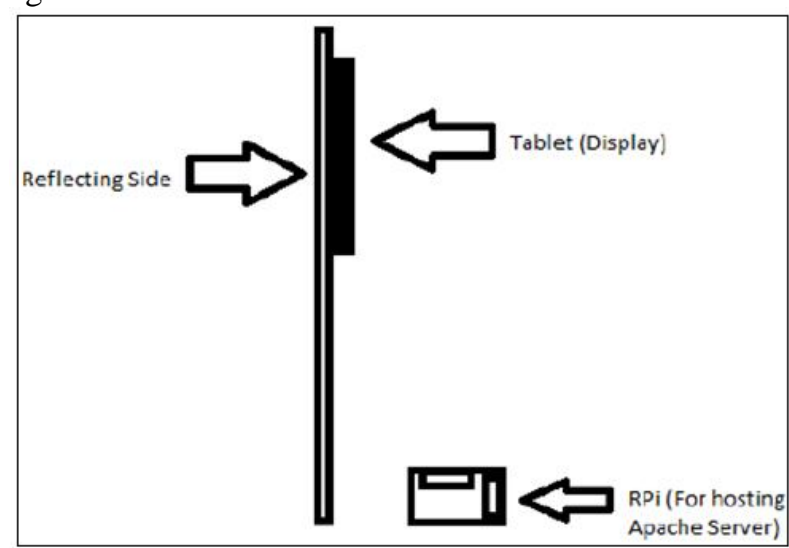

Figure 2: Hardware Design of Smart Mirror

\subsection{Software Design}

The design of the control software for the user was focused on 4 applications. These applications include weather outlook, google calendar, personal greeting and rss news feed. The GUI for of these applications was developed with a reasonably simple look and feel[7][8]. And every one the interface components were made to get on one single interface page positioned in exceedingly comfortable viewing places. The weather application was accustomed get the local weather outlook of this and for the upcoming days till per week.

The GUI was designed with a straightforward Helvetica font style and intuitive icons arranged within the list view. This complete application was designed with an easy pulling of information employing a unique APPID key. The APPID were grabbed from free OPENWEATHERMAP.API service into the applying and saved into the local variables of the config file used for the GUI design (Ref. Figure 3).

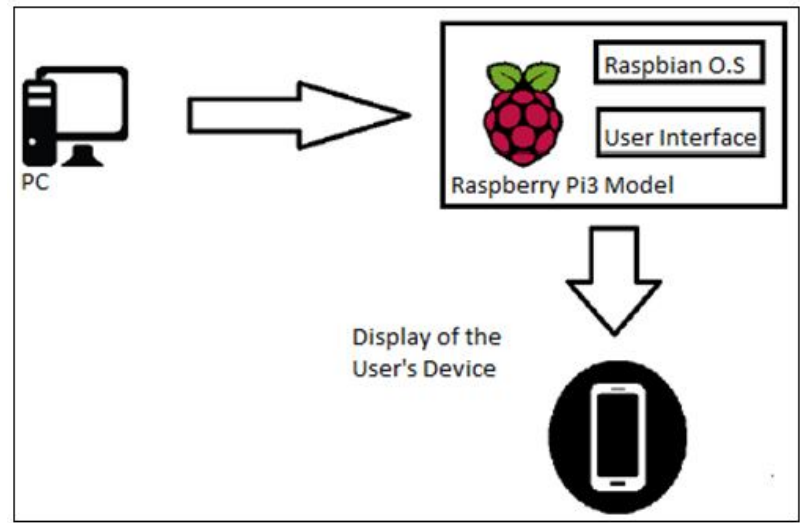

Figure 3: System Architecture of Smart Mirror
The calendar app was designed to produce the user's events for the present day [9]. The app was made to call the user's "iCal" file which was linked to the user's unique *.ics custom calendar link obtained from the calendars like Google, Apple, and others.

The URL link to the file was called from the smart mirror application. The smart mirror was made to call ASYNC tasks file from the corresponding links because the background process [10][11]. Once the files were downloaded, they got opened and parsed for to display the precise data. The custom parser was analyzed and also the date information was extracted with the event's time and outline. This data was appended in an orderly manner into the local vector variables for to urge the organized display within the GUI. The RSS news feeds has been pulled the most recent news, stories and headlines from NYTIMES news site. The RSS reader was made to tug the RSS data provided by NYTIMES. The local variables were set up to query the RSS URL and slice the required string for the formatted display.

\section{SETTING UP THE SMART MIRROR WITH GUI DESIGN}

\subsection{Foreground and Background Task Processing}

The raspberrypi is the heart of the complete system, acting with the web server and also serves the requirements of the whole model [12][13]. Supported the sort of interaction done by the user the following piece of data were pulled and displayed on the GUI (Figure.4).

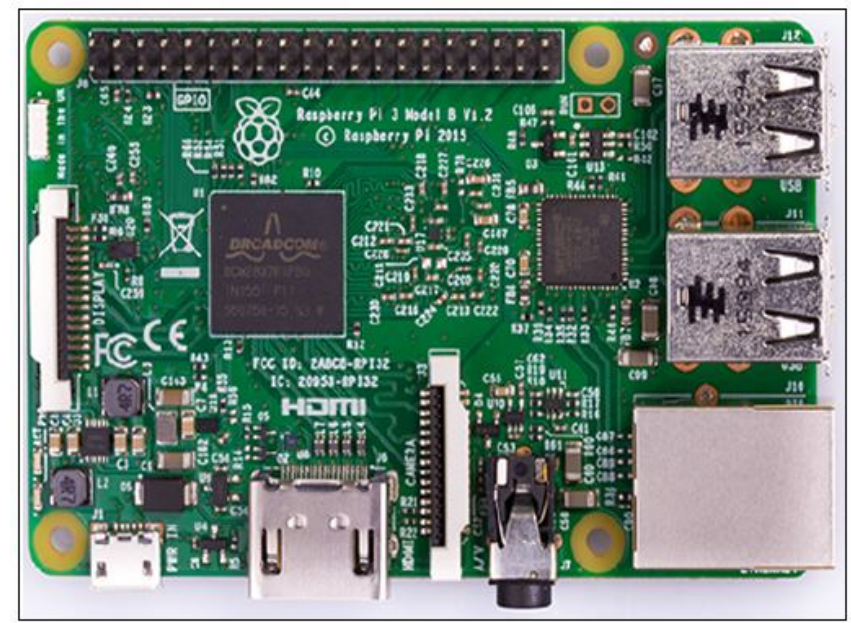

Figure 4: Raspberry Pi3 Model B kit

\subsection{User Interface and GUI Design}

The programme and GUI design were specifically designed for the necessities of the layman user (Figure 5,6). The GUI was enriched with the visually appealing effects to create sense, be unobtrusive, and simple to read. The programme 
was given the range of options for the user to interact in an intuitive way. This work discusses procedure of planning and building up a keen control framework that is utilized for controlling the smart mirror. An android application was created which has easy to understand Graphical Interface and it makes advantageous for client to associate with keen framework. A wifi across cloud framework was utilized for association of application and control framework which has snappy reaction to users inputs. The shading temperature and luminance was constrained by RGB RED PANNEL light. Inverter type climate control system can be controlled utilizing PDA application and for this reason an IR Remote module controller framework was assembled. The smart mirror was made to function through manual controlling mode for the convenience of the user.

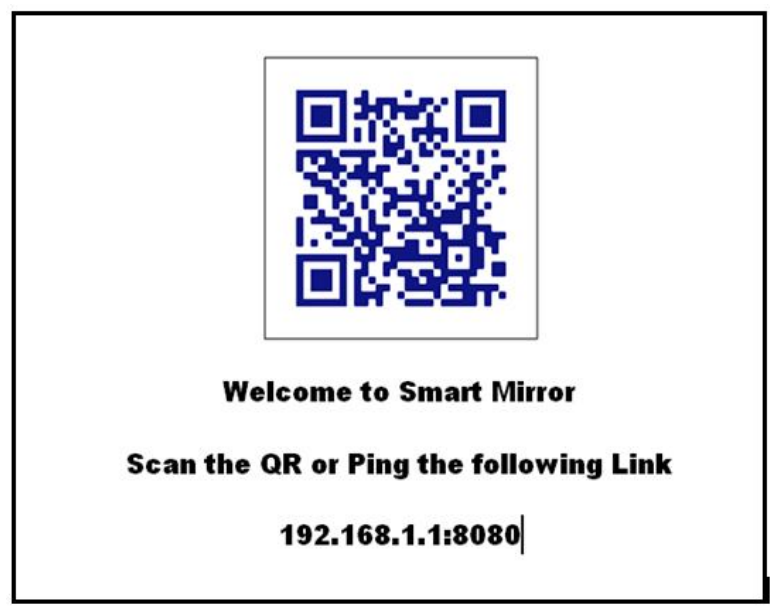

Figure 5: Welcome Screen including IP for Remote access

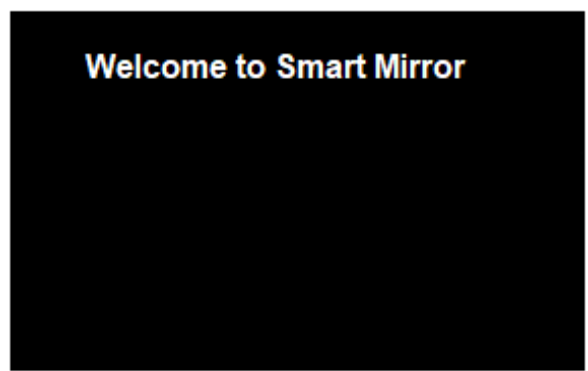

Figure 6: Welcome Screen without IP for Remote access

The commercial products have got to be developed with the utmost consumer usability, by following all the desired standards in terms of both functional and non-functional design requirements (Figure 7).

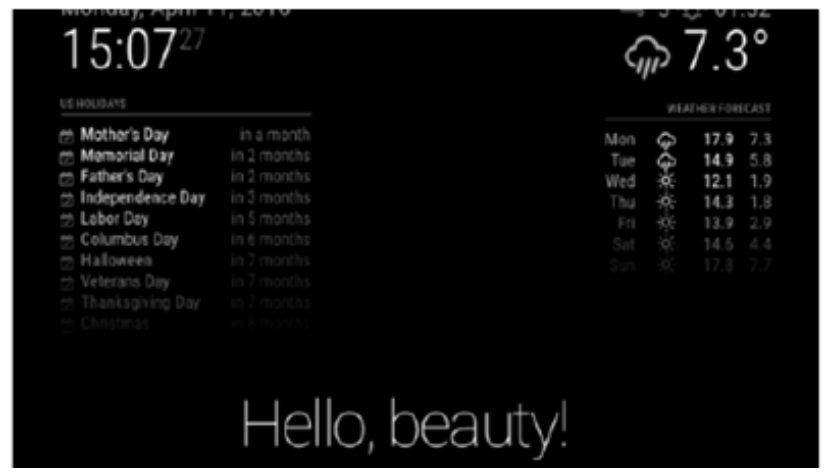

Figure 7: Home Page View with Head lines

As stated earlier, all the applications including the clock were made visibly available to the user and capable of sensing the user actions [15]. A flat, elegant and straightforward design with bold font was made to appearing on the top-left, top-right edges, center, left and bottom of the mirror in an exceedingly vertical view layout. Refer the screenshots for the UI layout below (Figure $8 \& 9$ ).

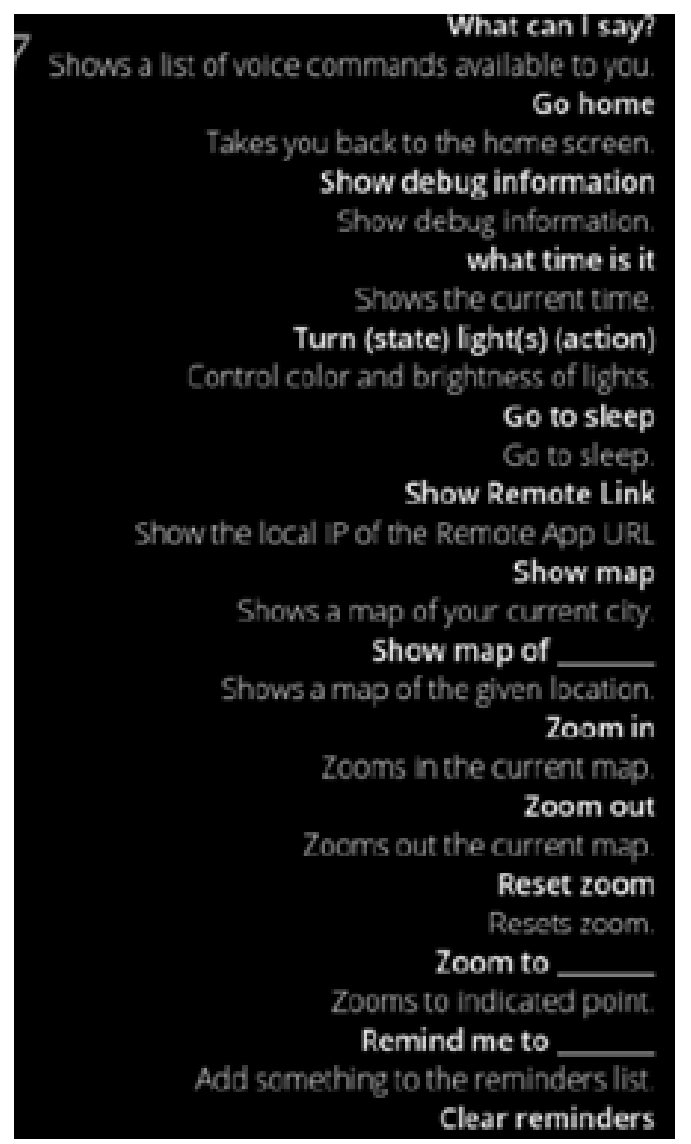

Figure 8: List of Commands 


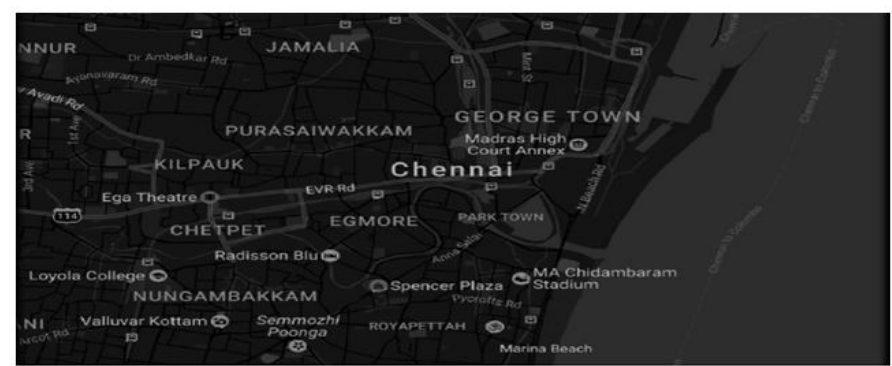

Figure 9: Map Application View

\subsection{Setting Up the Raspberry Pi3}

We have used Raspberry Pi 3 Model B and a HDMI compatible monitor: The Raspberry Pi was connected to a display. A USB keyboard and mouse was connected with the Raspberry Pi to control and configure the device [16][17]. The Raspberry Pi's operating system was installed on a MicroSD card. The recommended storage size for the raspbian based device is $8 \mathrm{~GB}$ maximum [18].

The Raspberry Pi was powered through the micro USB port with a $2.5 \mathrm{~A}$ of power adapter. Hence we have installed Raspbian version 3 and an image writer software (win32 disk manager). The raspberry $\mathrm{Pi} 3$ is that the third generation kit with $1.25 \mathrm{GHz} 64$ bit quad-core CPU. it's Bluetooth, wireless LAN, HDMI, Ethernet and other interfacing slots. Raspbian is that the debian based package for Raspberry pi. The Raspbian is acting on PIXEL ( $\mathrm{Pi}$ Improved $\mathrm{X}$ windows Environment Light). It also has LXDE desktop and window manager. The putty is an opensource terminal emulator that supports many protocols like SSH, SCP, Telnet, Rlogin. This putty software was accustomed control the raspberry pi without monitor [19].

The new Pi 3 consists of ARM CPU, compatible with remote LAN and Bluetooth interfaces. The Pi3 comes with the higher timed GPU. The two model B sheets are fundamentally the same as - every one of the ports are in a similar place. The GPIO header is used to control CPU, memory and USB controller chips [20]. Indeed, even cases intended for the Pi2-Model B are completely perfect with the new Pi 3, one might need to avoid metal cases to shield the Wi-Fi radio wire if there should arise an occurrence of the Pi 3 [21][22].

There are a couple of inconspicuous contrasts, The micro-SD card opening on the Pi 3 which is presently grinding based as opposed to the spring stacked push-push type utilized until presently. It is a notable aspect present with the Pi3 implementation [23]. In Pi3, the U16 control controller chip [24] has been covered with thicker paint to avert resetting the board because of xenon camera flashes. The GPIO header was used to receive the data from the remote node.

\subsection{Test Configuration}

The tests performed relied upon normal utilization situations. These include just utilizing the masses up one next to the opposite and taking estimations of your time, speed, execution and temperatures while playing out similar assignments in indistinguishable setups. The new Pi 3 Model $\mathrm{B}$ can get quite hot, within the wake of stacking the CPU at $100 \%$ for some minutes it absolutely was seen that the middle temperature surpassed 80 degrees Celsius, so appears that including some cooling components is key. Two warmth sinks, sourced from a GPU cooling unit, on the SoC itself and on the microchip on the other side of the board. The Raspberry Pi2 SoC will make sure the stable temperatures in order that a radiator isn't required as a rule.

\subsection{OS Installation and Time Taken to Boot}

The principal test was obviously introducing the standard Raspbian based environment which took very nearly 15 minutes on the Pi 2 and nearly 13 minutes on the Pi 3 . It is graphically shown in the below bar chart.

\subsection{OpenGL Performance}

The Open-Gl performance test was conducted to test the speed for the 3D rendering. The plateau utils bundle was introduced. The glx gears was utilized on the Pi's to test the sting rates that both the models would convey. The RPi2 oversaw about 26 FPS while the RPi3 achieved a traditional of 38 FPS, which is an expansion of over $49 \%$. The driving force was empowered from rasbpi-config within the advanced choices of the GL Driver segment. On the opposite hand the road data overlay $=\mathrm{vc} 4-\mathrm{kms}-\mathrm{v} 3 \mathrm{~d}$ is included into/boot/config.txt. The comparative study was made on the basis of data availed in online open source platform and the same is showcased below.

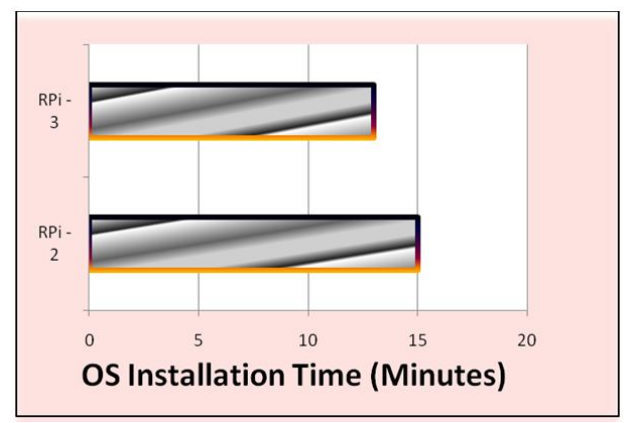

Figure 10: Comparison of Installation time between the different Raspbian implementations 


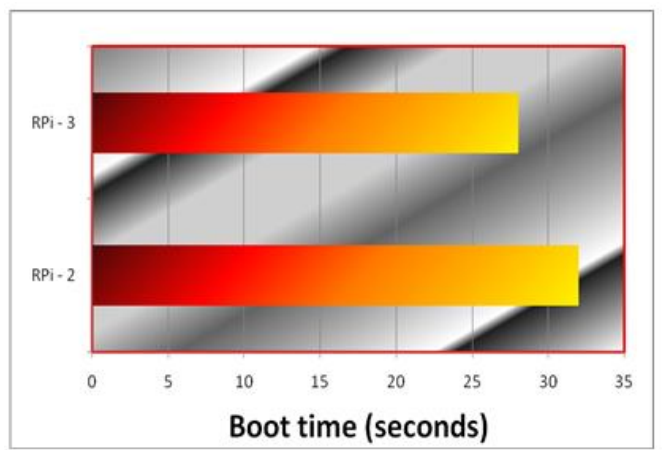

Figure 11: Comparison of Boot time between Different Raspbian variants

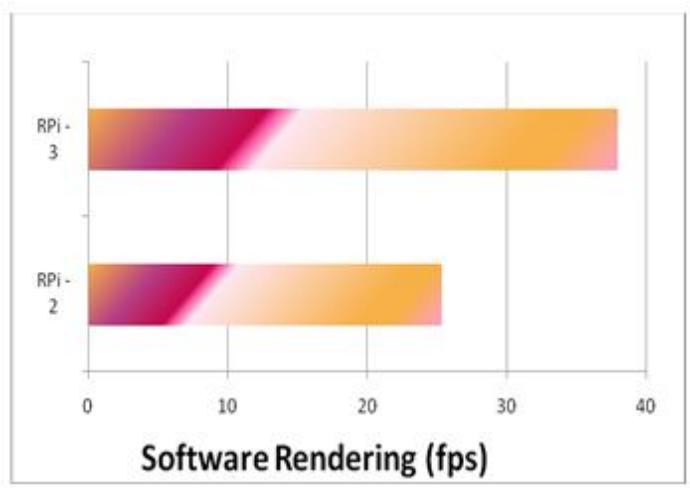

Figure 12: Comparison of software rendering between Raspbian 2 and 3

\section{PERFOMANCE EVALUATION}

The generated information was exchanged over the nearby system between a Windows 7 machine and each of the smart mirror implementation with Pi2 and Pi3. Every test was played out a few times for consistency and after that a normal esteem was determined while dispensing with far away readings. In all experiments just the separate interface was set dynamic to dispense with conceivable differences. In the principal test the speed of the interfaces were analyzed with LAN Speed Test, which mimics duplicating records starting with one PC then onto the next over a Windows organize, setting the document size to 50,100 and 200MB. To check the LAN exchange speed, the accompanying steps were done:

- Enable sharing.

- Created an envelope to share.

- And then adjusted the arrangement document of the Samba customer.

With the 150MbpsWi-Fi USB connector it appears that qualities had recognizable varieties on the Pi 3 and $\mathrm{Pi}$ 2. This time tests were performed with CPU stack to check the reaction time for every boards in different situations. The tests were conducted in a regular interval 20 seconds interim) for the readings to be more steady. The data download and sending were tried by considering every PC as either a server or a customer.

\subsection{Overall Performance}

The generated information was exchanged over the nearby system between a Windows 7 machine and every of the smart mirror implementation with Pi2 and Pi3. Every test was played out some times for consistency and at the moment a standard esteem made up our minds while dispensing with secluded readings. In all the above said experiments the separate interface was set dynamic to dispense with conceivable differences. within the principal test the speed of the interfaces were analyzed with LAN Speed Test, which mimics duplicating records starting with one PC then onto the following over a Windows organize, setting the document size to 50, 100 and $200 \mathrm{MB}$. It is evident that the overall performance of $\mathrm{RPi}-3$ is better over the other processors. The following table 1 replicates the same as above said inferrence.

Table 1: Performance analysis of RPi2 and RPi3

\begin{tabular}{|c|c|c|}
\hline $\begin{array}{c}\text { Performance } \\
\text { Analysis }\end{array}$ & RPi - 2 & RPi - 3 \\
\hline OS Installation & 15 minutes & 13 minutes \\
\hline Boot time & 32 seconds & 28 seconds \\
\hline Software Rendering & $25.4 \mathrm{fps}$ & $38 \mathrm{ps}$ \\
\hline
\end{tabular}

\section{CONCLUSION}

Thus the design of a novel smart mirror was made and the futuristic technological combo IoT and AI facilitates the user to interact in a highly efficient and effective way. The controlling functionalities of the SM is implemented with Raspberry Pi3 Model B. version 2 and 3. The functionalities and UIs' were expanded to control the house-hold devices. The smart mirror display is enhanced through positioning a flat monitor placed behind a 2 way mirror and the information displayed in the screen in a non-obstructive way so as to not interfere with traditional mirror function. The functional prototype is deployed after performing a detailed requirement analysis. Thus a next generation smart mirror framework was developed and analyzed in both the raspbian 2 and raspbian 3 . There have been different approaches for the implementation of Smart Mirror systems. It is possible to add novel functionality to control the household appliances remotely using the Smart Mirror, and also to add facial recognition to allow user authenticated actions. The Live news reports, traffic alerts and calender events may be displayed for the users to plan their day schedule on the go even in the car mirror. The potential for the further enhancement of this project is vast since different users may have their own requirements. Hence we decided to collect requirements from the people from different age groups and propose a novel smart mirror with an IoT based control of the home appliances. 


\section{REFERENCES}

[1] Li S, Da Xu L, Zhao S. The internet of things: a survey. Information Systems Frontiers. 2015 Apr 1;17(2):243-59.

[2] Maheshwari P, Kaur MJ, Anand S. Smart Mirror: A Reflective Interface to Maximize Productivity. International Journal of Computer Applications. 2017;166(9).

[3] Hossain MA, Atrey PK, El Saddik A. Smart mirror for ambient home environment.

[4] Fujinami K, Kawsar F, Nakajima T. AwareMirror: a personalized display using a mirror. International Conference on Pervasive Computing 2005 May 8 (pp. 315-332). Springer, Berlin, Heidelberg. https://doi.org/10.1007/11428572_19

[5] Hossain MA, Atrey PK, El Saddik A. Smart mirror for ambient home environment.

[6] Richardson M, Wallace S. Getting started with raspberry PI. "O'Reilly Media, Inc."; 2012 Dec 10.

[7] Prasad S, Mahalakshmi P, Sunder AJ, Swathi R. Smart surveillance monitoring system using Raspberry Pi and PIR sensor. Int. J. Comput. Sci. Inf. Technol. 2014 Jun;5(6):7107-9.

[8] Vujović V, Maksimović M. Raspberry Pi as a Sensor Web node for home automation. Computers \& Electrical Engineering. 2015 May 1;44:153-71.

[9] Richardson M, Wallace S. Getting started with raspberry PI. "O'Reilly Media, Inc."; 2012 Dec 10.

[10] Jain S, Vaibhav A, Goyal L. Raspberry Pi based interactive home automation system through E-mail. In Optimization, Reliabilty, and Information Technology (ICROIT), 2014 International Conference on 2014 Feb 6 (pp. 277-280). IEEE.

[12] Webster J, Fenton B, Stringer D, Bennetts B. On the synergy of boundary scan and emulation board test: a case study. InProc. of Board Test Workshop, Charlotte, USA 2003 Oct (p. 10).

[13] Senthilkumar G, Gopalakrishnan K, Kumar VS. Embedded image capturing system using raspberry pi system. International Journal of Emerging Trends \& Technology in Computer Science. 2014 Apr;3(2):213-5.

[14] Sandeep V, Gopal KL, Naveen S, Amudhan A, Kumar LS. Globally accessible machine automation using Raspberry pi based on Internet of Things. In Advances in Computing, Communications and Informatics (ICACCI), 2015 International Conference on 2015 Aug 10 (pp. 1144-1147). IEEE. https://doi.org/10.1109/ICACCI.2015.7275764

[15] Vujovic V, Maksimovic M. Raspberry Pi as a Wireless Sensor node: Performances and constraints. In Information and Communication Technology, Electronics and Microelectronics (MIPRO), 2014 37th International Convention on 2014 May 26 (pp. 1013-1018). IEEE.

[16] Ibrahim M, Elgamri A, Babiker S, Mohamed A. Internet of things based smart environmental monitoring using the Raspberry-Pi computer. InDigital Information Processing and Communications (ICDIPC), 2015 Fifth International Conference on 2015 Oct 7 (pp. 159-164). IEEE.

[17] Sundaram GS, Patibandala B, Santhanam H, Gaddam S, Alla VK, Prakash GR, Chandracha SC, Boppana S, Conrad JM. Bluetooth communication using a touchscreen interface with the Raspberry $\mathbf{P i}$. InSoutheastcon, 2013 Proceedings of IEEE 2013 Apr 4 (pp. 1-4). IEEE.

[18] Dudas R, VandenBussche C, Baras A, Ali SZ, Olson MT. Inexpensive telecytology solutions that use the Raspberry Pi and the iPhone. Journal of the American Society of Cytopathology. 2014 Jan 1;3(1):49-55.

[19] Pereira V, Fernandes VA, Sequeira J. Low cost object sorting robotic arm using Raspberry Pi. InGlobal Humanitarian Technology Conference-South Asia Satellite (GHTC-SAS), 2014 IEEE 2014 Sep 26 (pp. 1-6). IEEE.

[20] Zhao CW, Jegatheesan J, Loon SC. Exploring iot application using raspberry pi. International Journal of Computer Networks and Applications. 2015 Jan;2(1):27-34.

[21] Kochláň M, Hodoň M, Čechovič L, Kapitulík J, Jurečka M. WSN for traffic monitoring using Raspberry Pi board. In Computer Science and Information Systems (FedCSIS), 2014 Federated Conference on 2014 Sep 7 (pp. 1023-1026). IEEE

[22] ZA Shaffiei and N Abdullah, Addressing Factors Towards Human-Centered Technology, International Journal of Advanced Trends in Computer Science and Engineering, Volume 8, No.1.6, 2019,368 - 376 https://doi.org/10.30534/ijatcse/2019/5481.62019

[23] Mohammed Abbas Fadhil Al-Husainy and Bassam Al-Shargabi, Secure and Lightweight Encryption Model for IoT Surveillance Camera, International Journal of Advanced Trends in Computer Science and Engineering, ,Volume 9 No.2, March - April 2020, 1840 $-1847$. https://doi.org/10.30534/ijatcse/2020/143922020

[24] Nguyen HuyBinh, Use of Information Technology in Crime Investigation, International Journal of Advanced Trends in Computer Science and Engineering, Volume 9 No.2, March - April 2020, 1909 $-1912$. https://doi.org/10.30534/ijatcse/2020/154922020 